\title{
Valence Force Constants of Carbonyl Bonds in Naphthaldehydic Compounds as Chemical Binding Correlation Parameters
}

\author{
Victor Vargas, Ernesto Clavijo, and Raul G. E. Morales* \\ Department of Chemistry, Faculty of Sciences, University of Chile, Santiago, Chile
}

Z. Naturforsch. 40a, 927-931 (1985); received June 4, 1985

Valence force constants of carbonyl bonds in naphthaldehydic compounds were estimated by means of a point-charge model using a CNDO calculation. Experimental frequencies, in a solution of carbon tetrachloride, were analyzed in terms of the substituent effect. Vibrational assignments of bands which are sensitive to the substituents are proposed.

\section{Introduction}

The relative stabilities of various conformations of substituted naphthalenes and aromatic aldehydes have been deduced from several semiempirical and experimental methods [1-5]. However, to our knowledge, normal coordinate analysis, or the characterization of the vibrational potential function of these large molecules, have not been published up to now.

In order to analyze the substituent effect on the carbonyl bond in naphthaldehydic compounds, we have used a point-charge model developed by Kosmus [6]. The valence force constants of the carbonyl bond are studied by means of the substituent effect across the aromatic system.

The Kosmus formulation, a simple method to discuss bond properties at the equilibrium position, allows us to estimate the electronic contributions to the force constants. These valence force constants calculated from computational $\mathrm{CNDO} / 2$ data have been shown to be appropriate to describe various molecular systems [7].

The valence force constants, $f(\mathrm{OC})$, of a bond $\mathrm{O}-\mathrm{C}$ according to Kosmus's work (6) is given by the equation

$f(\mathrm{OC})=\frac{\left(Z_{\mathrm{O}}-2\right)\left(2 Z_{\mathrm{O}}-Q_{\mathrm{O}}\right)}{Z_{\mathrm{O}} R^{3}}\left(8 B(\mathrm{OC})-q_{\mathrm{C}}\right)$,

* Visiting Professor at the Universidad Autonoma de Madrid, October-December 1984.

Reprint requests to Dr. R. G. E. Morales, Universidad de Chile, Facultad de Ciencias, Departamento de Quimica, Casilla 653, Santiago, Chile. where $Z_{\mathrm{O}}$ is the charge on $\mathrm{O}$ used in the $\mathrm{CNDO} / 2$ calculation, $Q_{\mathrm{O}}$ is the gross charge on $\mathrm{O}, q_{\mathrm{C}}$ is the net charge on the aromatic carbon atom, $R$ is the $\mathrm{C}-\mathrm{O}$ bonding length and $B(\mathrm{OC})$ is the bond index calculated from the Wiberg bond index $(W(\mathrm{OC}))$. The bond index $B(\mathrm{OC})$ for single bonds is equal to $W(\mathrm{OC})$, whereas for double bonds (i.e., for carbonyl bond) is given by:

$$
B(\mathrm{OC})=W(\mathrm{OC})+(1 / 2)\left\{W_{\pi}(\mathrm{OC})\right\}^{1 / 2} .
$$

Whereas a lot of data exits for the simplest aldehydic systems, only few works have been reported for naphthaldehydic compounds. In this paper, we report vibrational force constants for these compounds calculated by means of the $\mathrm{CNDO} / 2$ quantum chemical method. An assignment of a vibrational frequency mode, sensitive to the substituent effect, is also proposed.

\section{Experimental}

1-Naphthaldehyde (1-NA); 4-methoxy,1-naphthaldehyde (4,1-MNA); 2-methoxy,1-naphthaldehyde (2,1-MNA); 2-naphthaldehyde (2-NA) and 3-methoxy,2-naphthaldehyde (3,2-MNA) were obtained from Aldrich Chem. Co. 2-Hydroxy,1-naphthaldehyde (2,1-HNA); 3-hydroxy,2-naphthaldehyde (3,2-HNA) and 1-hydroxy,2-naphthaldehyde (1,2HNA) were synthetized by Professor A. Alvarado at Academia Superior de Ciencias Pedagógicas de Santiago de Chile.

All compounds were purified by distallation of fractional sublimation before their use. 
Infrared spectra were registered in a Perkin Elmer 621 spectrophotometer, using cells with sodium chloride windows and a path length of about $0.015 \mathrm{~mm}$ for the pure liquids. Samples of the naphthaldehydes in a solution of carbon tetrachloride (path length $0.5 \mathrm{~mm}$ ) were prepared at room temperature, and the $\mathrm{KBr}$ disk technique was used with the solid samples. Carbon tetrachloride was Merck, Uvasol, spectroscopic grade.

\section{Computational Method}

A CNDO/2 computational programme [8] was employed and standard geometries were obtained from the literature $[1,2,8]$. Angle and bond length optimizations for $\mathrm{C}=\mathrm{O}$ and substituent groups were carried out [9].

An intramolecular hydrogen bond was assumed to exist in the hydroxynaphthaldehyde compounds, and all molecular structures were considered to be planar.

\section{Results and Discussion}

Table 1 shows both the calculated gross and net charge on the $\mathrm{O}$ and $\mathrm{C}$, and the Wiberg bond indices in these carbonyl compounds. The calculated Kosmus force constants $f(\mathrm{OC})$ and experimental values $f^{\prime}(\mathrm{OC})$ can be compared in Table 2. In the latter the carbonyl bond is considered as a pseudodiatomic system $\left(f^{\prime}(\mathrm{OC})=4.040 \cdot 10^{-6} v^{2}(\mathrm{mdyn} /\right.$ $\AA)$ ). Obviously, this rough calculation has been considered as a simple approach, assuming a negligible coupling between the vibrations of the $\mathrm{C}=\mathrm{O}$ group and the other vibrations of the molecule. Therefore these values of force constants should be considered as upper values which arise from normal coordinate analysis, due to the electronic conjugation between the double bond and the substituted aromatic ring. A good agreement can be found between these two sets of force constants, where $f(\mathrm{OC})$ is about $10 \%$ lower than $f^{\prime}(\mathrm{OC})$.

A relationship between the square root of $f(\mathrm{OC})$, for these carbonyl compounds, and the $\mathrm{C}=\mathrm{O}$ stretching frequency would permit us to obtain information about the effect of the substituent on the $\pi$ nature of the carbonyl bond. Figure 1 shows a good linear correlation between these experimental fre-
Table 1. Length, charge and Wiberg indices of the carbonyl bond in the naphthaldehydic compounds.

\begin{tabular}{llllll}
\hline Compound & $R(\mathrm{~A})$ & $Q_{\mathrm{O}}$ & $q_{\mathrm{C}}$ & $W_{\sigma}(\mathrm{OC})$ & $W_{\pi}(\mathrm{OC})$ \\
\hline 1-NA & 1.265 & 6.254 & 0.220 & 1.030 & 0.840 \\
4,1-MNA & 1.265 & 6.268 & 0.226 & 1.031 & 0.826 \\
2,1-MNA & 1.265 & 6.270 & 0.233 & 1.031 & 0.823 \\
2,1-HNA & 1.275 & 6.299 & 0.253 & 1.026 & 0.775 \\
2-NA & 1.265 & 6.246 & 0.216 & 1.030 & 0.855 \\
3,2-MNA & 1.265 & 6.255 & 0.232 & 1.039 & 0.846 \\
3,2-HNA & 1.270 & 6.272 & 0.248 & 1.027 & 0.811 \\
1,2-HNA & 1.270 & 6.297 & 0.258 & 1.028 & 0.782 \\
\hline
\end{tabular}

Table 2. Frequencies, valence force constants $(f)$ and approximate diatomic force constants $\left(f^{\prime}\right)$ of the carbonyl bonds in naphthaldehydic compounds.

\begin{tabular}{llcl}
\hline Compound & $v\left(\mathrm{~cm}^{-1}\right)$ & $f(\mathrm{OC})$ & $f^{\prime}(\mathrm{OC})$ \\
\hline 1-NA & 1699 & 10.48 & 11.66 \\
4,-MNA & 1690 & 10.39 & 11.54 \\
2,1-MNA & 1681 & 10.37 & 11.42 \\
2,1-HNA & 1642 & 9.808 & 10.89 \\
2-NA & 1702 & 10.57 & 11.70 \\
3,2-MNA & 1693 & 10.55 & 11.58 \\
3,2-HNA & 1667 & 10.15 & 11.23 \\
1,2-HNA & 1648 & 9.966 & 10.97 \\
\hline
\end{tabular}

$f^{\prime}(\mathrm{OC})=4.040 \cdot 10^{-6} v^{2}(\mathrm{mdyn} / \AA)$.

$v$ in solution of carbon tetrachloride.

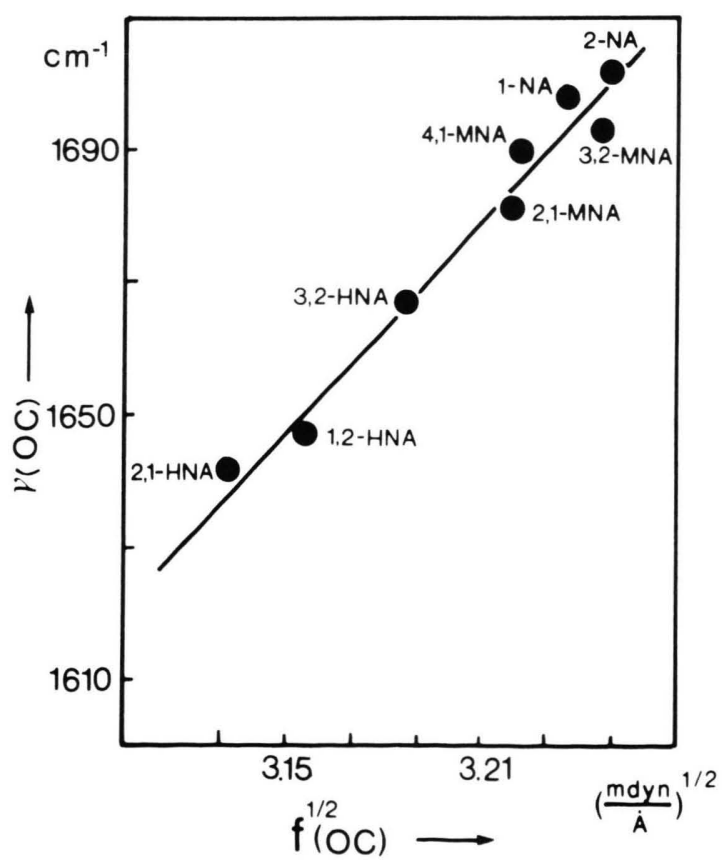

Fig. 1. Relation between force constant and stretching frequency for $\mathrm{CO}$ bonds in the naphthaldehydic compounds. 
Table 3. $2 p(z)$ Orbital coefficients from density matrix $\mathrm{CNDO} / 2$.

\begin{tabular}{llllllll}
\hline \multirow{2}{*}{$\begin{array}{c}\text { Com- } \\
\text { pound }\end{array}$} & \multicolumn{2}{l}{ Atomic Center* } \\
\cline { 2 - 8 } & 1 & 2 & 3 & 4 & 5 & 6 & 7 \\
\hline 1-NA & 1.037 & 0.961 & 1.014 & 0.969 & 0.829 & 1.234 & \\
4,1-MNA & 1.082 & 0.931 & 1.094 & 0.917 & 0.822 & 1.256 & 1.886 \\
2,1-MNA & 1.107 & 0.914 & 1.066 & 0.940 & 0.821 & 1.259 & 1.891 \\
2,1-HNA & 1.240 & 0.864 & 1.069 & 0.929 & 0.779 & 1.329 & 1.901 \\
2-NA & 0.980 & 1.015 & 0.982 & 1.101 & 0.838 & 1.213 & \\
3,2-MNA & 0.945 & 1.060 & 0.941 & 1.092 & 0.826 & 1.232 & 1.895 \\
3,2-HNA & 0.937 & 1.081 & 0.905 & 1.106 & 0.790 & 1.290 & 1.915 \\
1,2-HNA & 0.866 & 1.127 & 0.951 & 1.076 & 0.778 & 1.326 & 1.896
\end{tabular}

* Cf. Figure 2.

Table 4. Length, charge, Wiberg indices, and force constants of the carbon-oxygen bond between the aromatic carbon and the oxygen of the substituent $(f(\mathrm{OCar}))$ in naphthaldehydic compounds. $f(\mathrm{OCar})$ in mdyn/Å.

\begin{tabular}{lllllll}
\hline $\begin{array}{l}\text { Com- } \\
\text { pounds }\end{array}$ & $R(\mathrm{~A})$ & $Q_{\mathrm{O}}$ & $q_{\mathrm{C}}$ & $\begin{array}{l}W_{\sigma} \\
(\mathrm{OC})\end{array}$ & $\begin{array}{l}W_{\pi} \\
(\mathrm{OC})\end{array}$ & $\begin{array}{l}f \\
\text { (OCar) }\end{array}$ \\
\hline 4,1-MNA & 1.366 & 6.208 & 0.201 & 0.925 & 0.083 & 5.038 \\
2,1-MNA & 1.368 & 6.212 & 0.206 & 0.925 & 0.079 & 4.992 \\
2,1-HNA & 1.356 & 6.296 & 0.232 & 0.965 & 0.109 & 5.375 \\
3,2-MNA & 1.368 & 6.213 & 0.193 & 0.926 & 0.073 & 4.984 \\
3,2-HNA & 1.361 & 6.294 & 0.207 & 0.965 & 0.090 & 5.244 \\
1,2-HNA & 1.357 & 6.295 & 0.224 & 0.966 & 0.113 & 5.371 \\
\hline
\end{tabular}
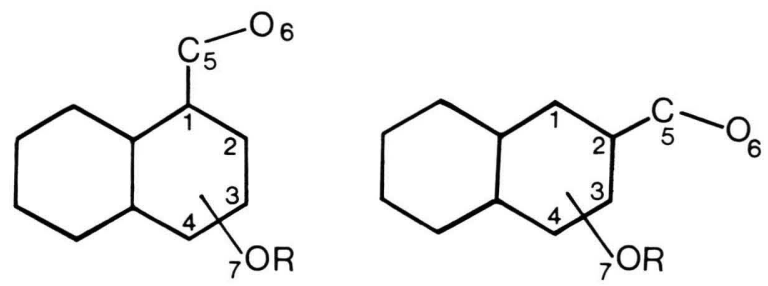

Fig. 2. Atomic center numeration in the two naphthaldehyde series under study (see Table III).

quencies in solution of carbon tetrachloride and the square root of the Kosmus force constant. The slope is $523.2\left((\mathrm{mdyn} / \AA)^{-1 / 2} / \mathrm{cm}^{-1}\right)$.

Other force constant studies based on semiempirical correlations [10] could be used in the present work in order to appreciate the accuracy of these $f(\mathrm{OC})$ values. However, our main interest rests in the expectation of the $\mathrm{CNDO} / 2$ calculation of the spectral shifts description due to the electronic distribution change induced by the substituent. Therefore, from the density matrix, the electronic correla- tion between the electron-donor and the electronacceptor groups through to the $2 \mathrm{p}(\mathrm{z})$ atomic orbitals permits us to observe the charge transfer which the substituent induces on the carbonyl bond, specifically on the oxygen atom (Table 3) [9]. In this way, the perturbation which the substituent introduces on the carbonyl bond frequency can be appreciated by means of the Wiberg bond indices, as well as charge densities (Tables 1 and 4). These two parameters were involved in the valence force constant calculation.

As can be seen from Figure 1, the two sets of naphthaldehyde compounds, the hydroxy- and the methoxy-derivatives series, are localized in two different spectral ranges. A good agreement was found between experimental and computational results. The additional increase of charge density on the oxygen atom of the $\mathrm{C}=\mathrm{O}$ group in the hydroxyderivative series is due to the charge transfer from the hydrogen of the hydroxyl group. At the same time, the decrease in covalent character of the $\mathrm{C}=\mathrm{O}$ bond is in good agreement with the diminution of the observed vibrational frequency. When the intramolecular hydrogen-bond was disrupted by a new conformation, with the hydroxyl group rotated 180 degrees, these computational results showed a similar behavior for hydroxy- and methoxy-substituents.

It is interesting to remark that the linear correlation in Fig. 1 is not dependent on the localization of the carbonyl and the substituent groups in the aromatic ring. Therefore the perturbation on the $\mathrm{C}=\mathrm{O}$ stretching essentially appears as an effect of the $\pi$-delocalization induced by the substituent on the aromatic ring, notwithstanding the 1-naphthaldehyde series localizes more charge on the oxygen atom of the $\mathrm{C}=\mathrm{O}$ group than the 2-naphthaldehyde series.

A good correlation was found between the valence force constants of the carbonyl bond, $f(\mathrm{OC})$, and the carbon-oxygen bond for the substituted naphthaldehydes, $f$ (OCar), as can be seen in Figure 3. Thus, by means of this relationship, the substituent can be considered as a charge regulator of the carbonyl bond.

Spectral band assignments of aromatic compounds are very difficult. At the same time, there is a lack of this type of assignments in the literature. However, ring spectral bands sensitive to substituent effect in this naphthaldehyde series could be tentatively assigned, considering the good linear corre- 


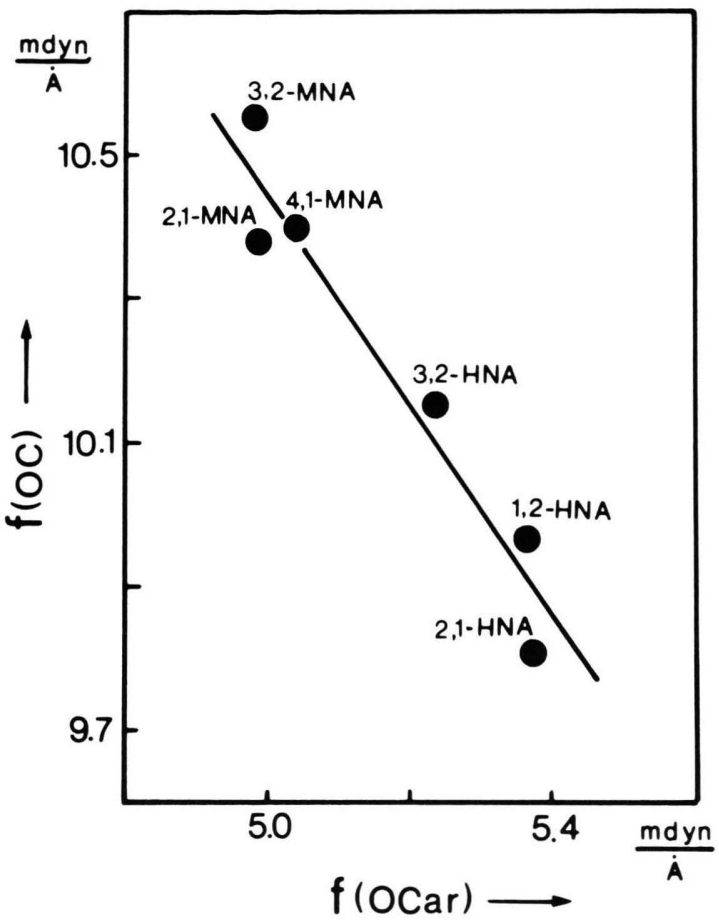

Fig. 3. Relation between force constant of the carbonyl bond $f(\mathrm{OC})$ and the aromatic carbon-oxygen of the substituent bond $f(\mathrm{OCar})$ for naphthaldehydic compounds.

\begin{tabular}{ll}
\hline $\begin{array}{l}\text { Com- } \\
\text { pounds }\end{array}$ & $v,\left(\mathrm{~cm}^{-1}\right)$ \\
\hline 4,1-MNA & \\
2,1-MNA & 1248 \\
2,1-HNA & 1246 \\
3,2-MNA & 1310 \\
3,2-HNA & 1248 \\
1,2-HNA & 1310 \\
\hline
\end{tabular}

Table 5. Ring band sensitive to substituent in the naphthaldehydic compounds.

a Solid samples.

b Liquid sample.

lation between the $f(\mathrm{OC})$ and $f(\mathrm{OCar})$ shown in Figure 3. Experimental frequencies of the ring bands sensitive to the substituent have been chosen in order to preserve a good linear relationship with the $f(\mathrm{OCar})$ values, in a similar way as shown in Fig. 1 (see Table 5). Thus, the semiempirical method developed in order to obtain this linear correlation (Fig. 4) has permitted us to give an additional criterium for assigning the experimental band data, such as the region and the shape of the spectral band in this kind of aromatic compound.

Finally, we have found that this methodology is suitable to describe the substituent effect and the

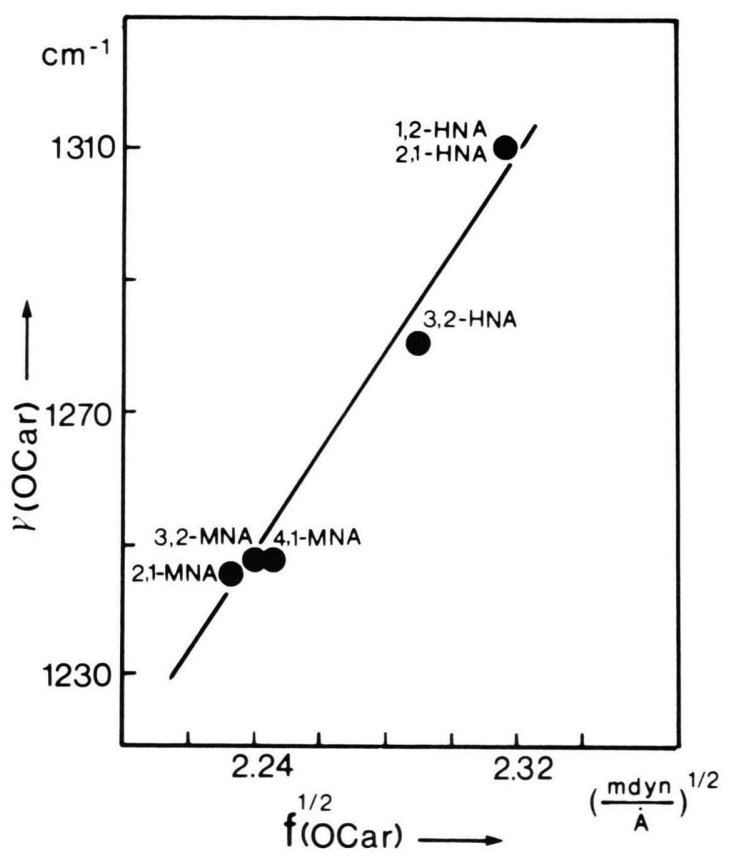

Fig. 4. Relation between force constant $f$ (OCar) and ring band frequency sensitive to substituent for naphthaldehydic compounds.

ring spectral band sensitive in benzaldehyde compounds [11]. At present, we are interested in extending these studied to other kinds of molecular systems involving charge transfer between electronacceptor and electron-donor groups in aromatic rings $(\mathrm{A}-\mathrm{Ar}-\mathrm{D})$.

\section{Acknowledgements}

This work has been supported by the Departamento de Desarrollo de la Investigación de la Universidad de Chile. We extend our acknowledgements to the Centro de Computación de la Universidad de Chile for generous amounts of free computer time.

One of us (R.G.E.M.) would like to express his thanks to the Ministerio de Educación y Ciencia de España for the fellowship awarded as Visiting Professor at the Universidad Autónoma de Madrid and the facilities and stimulating environment during the redaction of the present work. We also appreciate helpful comments and critical reading of the manuscript from Professors M. Campos and F. Uribe. 
[1] R. Benassi, D. Iarossi, U. Folli, L. Schetti, and F. Taddei, J. Chem. Sci. Perkin II, 228 (1981)

[2] S. R. Salman, Spectrochim. Acta 39 A, 1073 (1983); 40 A, 229 (1984)

[3] T. Schaefer, S. R. Salman, and T. A. Wildman, Can. J. Chem. 58, 2364 (1980).

[4] F. A. L. Anet and M. Ghiaci, J. Chem. Sci., Chem. Comm. 1979, 588.

[5] C. L. Cheng, R. J. W. Le Fevre, L. D. Ritchie, P. A. Goodman, and P. H. Gore, J. Chem. Soc. (B) 1971, 1198.
[6] W. Kosmus, Z. Naturforsch. 33 a, 1056 (1978).

[7] G. Diaz Fleming, W. Kosmus, and K. Kalcher, Z. Naturforsch. 36 a, 759 (1981).

[8] J. A. Pople and D. L. Beveridge, Approximate Molecular Orbital Theory, McGraw Hill, New York 1970.

[9] V. Vargas, Thesis, Universidad de Chile (1985).

[10] J. A. Ladd, W. J. Orville Thomas, and B. C. Cox, Spectrochim. Acta 20 A, 1771 (1964)

[11] R. G. E. Morales, V. Vargas, and E. Clavijo, Acta sud Amer. Quim. (to be published). 